\title{
BMJ Open Evaluating the efficacy of intranasal oxytocin on pain and function among individuals who experience chronic pain: a protocol for a multisite, placebo- controlled, blinded, sequential, within- subjects crossover trial
}

\author{
Joshua A. Rash (D) , ${ }^{1}$ Tavis S. Campbell, ${ }^{2}$ Lynn Cooper, ${ }^{3}$ David Flusk, ${ }^{4}$ \\ Aaron Maclnnes, ${ }^{5}$ Maryam Nasr-Esfahani, ${ }^{6,7}$ Anastasia A. Mekhael (D) , ${ }^{1}$ \\ Patricia A. Poulin, ${ }^{8,9,10}$ Magali Robert, ${ }^{6,7}$ Yanqing $\mathrm{Yi}^{11}$
}

To cite: Rash JA, Campbell TS, Cooper L, et al. Evaluating the efficacy of intranasal oxytocin on pain and function among individuals who experience chronic pain: a protocol for a multisite, placebo-controlled, blinded, sequential, within-subjects crossover trial. BMJ Open 2021;11:e055039. doi:10.1136/ bmjopen-2021-055039

- Prepublication history and additional supplemental material for this paper are available online. To view these files, please visit the journal online (http://dx.doi.org/10.1136/ bmjopen-2021-055039).

Received 03 July 2021 Accepted 11 August 2021

Check for updates

(C) Author(s) (or their employer(s)) 2021. Re-use permitted under CC BY-NC. No commercial re-use. See rights and permissions. Published by BMJ.

For numbered affiliations see end of article.

Correspondence to Dr Joshua A. Rash; jarash@mun.ca

\section{ABSTRACT}

Introduction Current treatments for chronic pain (eg, opioids) can have adverse side effects and rarely result in resolution of pain. As such, there is a need for adjuvant analgesics that are non-addictive, have few adverse side effects and are effective for pain management across several chronic pain conditions. 0xytocin is a naturally occurring hormone that has gained attention for its potential analgesic properties. The objective of this trial is to evaluate the efficacy of intranasal oxytocin on pain and function among adults with chronic pain.

Methods and analysis This is a placebo-controlled, triple-blind, sequential, within-subject crossover trial. Adults with chronic neuropathic, pelvic and musculoskeletal pain will be recruited from three Canadian provinces (British Columbia, Alberta and Newfoundland and Labrador, respectively). Enrolled patients will provide one saliva sample pretreatment to evaluate basal oxytocin levels and polymorphisms of the oxytocin receptor gene before being randomised to one of two trial arms. Patients will self-administer three different oxytocin nasal sprays twice daily for a period of 2 weeks (ie, $24 \mathrm{IU}$, $48 \mathrm{IU}$ and placebo). Patients will complete daily diaries, including standardised measures on day 1 , day 7 and day 14. Primary outcomes include pain and pain-related interference. Secondary outcomes include emotional function, sleep disturbance and global impression of change. Intention-to-treat analyses will be performed to evaluate whether improvement in pain and physical function will be observed posttreatment.

Ethics and dissemination Trial protocols were approved by the Newfoundland and Labrador Health Research Ethics Board (HREB \#20227), University of British Columbia Clinical Research Ethics Board (CREB \#H20-00729), University of Calgary Conjoint Health Research Ethics Board (REB20 \#0359) and Health Canada (Control \# 252780). Results will be disseminated through publication in peer-reviewed journals and presentations at scientific conferences.

Trial registration number NCT04903002; Pre-results.
Strengths and limitations of this study

The effect of oxytocin will be evaluated across different chronic pain presentations (ie, musculoskeletal, pelvic and neuropathic pain), and clinically relevant outcomes will be measured as recommended by the Initiative on Methods, Measurement and Pain Assessment in Clinical Trials.

- Two doses of oxytocin will be administered, to evaluate a dose-response relationship.

- The effect of oxytocin will only be evaluated over a 2-week period, precluding longer-term assessment.

- The $48 \mathrm{IU}$ dosing is the last course of treatment in both trial arms, which may introduce expectancy effects due to incomplete blinding at this dose.

- Saliva samples will be collected to measure basal oxytocin levels and polymorphisms of the oxytocin receptor gene as potential moderators of treatment effect.

\section{INTRODUCTION}

In 2011, the Institute of Medicine concluded that chronic pain, defined as pain that persists longer than 3 months or beyond the expected duration of healing, ${ }^{1}$ is a public health concern and should be treated as a disease itself. ${ }^{2}$ Nationally representative data indicates that $20 \%$ of Canadians over 18 years of age ${ }^{3}$ and $15 \%$ of children ${ }^{4}$ suffer with chronic pain. The prevalence of chronic pain increases with age. Approximately $65 \%$ of community dwelling seniors and $80 \%$ of older adults living in care facilities experience chronic pain. ${ }^{5}$

Currently available treatments for chronic pain rarely result in complete resolution of symptoms, ${ }^{6}$ and often do not produce 
concomitant improvements in physical and emotional functioning. ${ }^{7}$ For example, a 2018 meta-analysis reported that 1 in 8 patients respond to opioid medication with a mean $6.9 \mathrm{~mm}$ (on a 100-mm Visual Analogue Scale) reduction in pain, small improvement in physical function and no improvement in mental function. ${ }^{8}$ Moreover, current pharmacological treatments for pain, including opiates, are often addictive, associated with adverse effects, ${ }^{910}$ and have limited effectiveness in areas such as neuropathic pain. Given the gap between suffering and adequate pain management, there is a need for analgesics that are safe, non-addictive, have low adverse effect profiles and offer effective relief for a variety of painful conditions.

Intranasal oxytocin has gained increasing attention in recent years as a promising analgesic. ${ }^{11-14}$ Oxytocin is a neuropeptide that is produced in the hypothalamus, ${ }^{15}$ and released into the peripheral and central nervous system through independent pathways. ${ }^{16}$ Evidence from several sources, including our team, suggests that oxytocin may be a safe and effective method for pain management. ${ }^{12} 13$ Oxytocin may decrease pain sensitivity through three mechanisms $^{12}$ : (1) oxytocin is transported to an area involved in pain modulation, Laminae I, II and IV of the dorsal horn, through a hypothalamic-spinal projection. ${ }^{16}$ Approximately $35 \%$ of neurons in the dorsal horn contain oxytocin receptors that act to inhibit pain-carrying $A \delta$-fibres and C-fibres $^{17-19}$; (2) oxytocin binds to opioid receptors, and results in analgesic effects when administered to the periaqueductal grey, and effect that can be blocked with an opioid antagonist. ${ }^{20}{ }^{21}$ Furthermore, analgesic effects of endogenous and exogenous oxytocin can be blocked by the opioid antagonist naloxone ${ }^{22}$; and (3) oxytocin may decrease pain sensitivity by improving mood, reducing anxiety and buffering stress given that the induction of negative emotions are associated with heightened pain ${ }^{23-25}$ and autonomic arousal. ${ }^{24}$ In an informative controlled trial, intranasal administration of oxytocin in men resulted in greater calmness, less anxiety and a trend toward lower cortisol during the Trier Social Stress Test. ${ }^{26}$

Preliminary evidence suggests that oxytocin may be an effective adjuvant analgesic that is applicable to a broad patient population. Our team published a systematic review of the effect of oxytocin on pain in animals and humans. ${ }^{12}$ Oxytocin had a reliable effect as defined by increasing pain tolerance in 29 out of 33 animal studies reviewed. This effect was large (standard mean difference $=2.28$ ), and persisted across central and peripheral modes of administration and various noxious stimuli (eg, heat, electric and chemical). ${ }^{12}$ Results from research into the association between oxytocin and pain in humans have been variable due to methodological heterogeneity. For example, two studies have assessed associations between oxytocin and pain using experimental pain procedures in healthy adults, reporting a decrease in pain sensitivity to finger prick, ${ }^{27}$ and no difference in pain unpleasantness to electric shock. ${ }^{28}$ Interpreting these results is difficult due to methodological concerns, including insufficient sample size, one-dimensional pain measurement ${ }^{28}$ and use of a poorly described finger prick pain procedure. ${ }^{27}$ Our team conducted the first methodologically rigorous placebo-controlled, blinded, within-subjects crossover trial evaluating the effect of intranasal oxytocin on acute pain. ${ }^{29}$ We observed clinically meaningful effects of oxytocin on pain, particularly neuropathic indicators. With regard to chronic pain, individuals experiencing chronic back pain, ${ }^{30}$ headache, ${ }^{31}$ constipation ${ }^{32}$ and colon pain ${ }^{33}$ have reported lower sensitivity to pain following the administration of oxytocin. Confidence in the association between oxytocin and chronic pain has been difficult to discern due to limitations in study designs, including selection of an insufficient control condition, ${ }^{31}$ use of an intrathecal punch delivery method that likely confounded pain assessment ${ }^{30}$ administration of oxytocin peripherally without verification of influence in the central nervous system ${ }^{33}$ or inadequate statistical power to detect meaningful effects. ${ }^{32}{ }^{34}$ Furthermore, no trial to date has assessed all clinically relevant outcomes endorsed by the Initiative on Methods, Measurement and Pain Assessment in Clinical Trials (IMMPACT) —an international group of experts that develop recommendations to improve the design, execution and interpretation of clinical trials of treatments for pain. ${ }^{35}$

Given the heterogeneity of extant trials, we propose a methodologically rigorous trial evaluating the efficacy of intranasal oxytocin on pain and function among men and women with chronic pain that evaluates clinically relevant outcomes endorsed by the IMMPACT ${ }^{35}$ Potential mediators and moderator of treatment effects will be assessed using basal oxytocin levels and polymorphisms of the oxytocin receptor gene measured using salivary assays given that: (1) chronic pain patients exhibit low basal oxytocin levels relative to controls, ${ }^{303637}$ and may reflect underlying abnormality in the oxytocinergic system ${ }^{12}$ and (2) relative to those with an rs53576 A allele, individuals with a rs53576G/G oxytocin receptor genotype show reduced amygdala, ${ }^{38}$ neuroendocrine $^{26}$ and stress reactivity across a range of contexts, ${ }^{39} 40$ and report greater benefit from social support ${ }^{41}$ following oxytocin administration. This represents a movement toward precision medicine.

\section{Research questions}

We will evaluate the efficacy of intranasal oxytocin when used as an adjuvant treatment (ie, in addition to usual therapies) for improving pain and function (physical and emotional) among men and women with chronic neuropathic, musculoskeletal or pelvic pain.

\section{Primary hypotheses}

Relative to placebo, patients will report greater improvement in: (1) pain intensity and (2) physical function measured using the Brief Pain Inventory-Short Form (BPI-SF) following a 2-week course of twice daily 24IU or 48 IU intranasal oxytocin administration.

\section{Secondary hypotheses}

Patients will report improvement in emotional function, sleep and global impression of change following intranasal oxytocin administration relative to placebo. 


\section{Exploratory questions}

Potential mediators and moderator of treatment effects will be assessed using basal oxytocin levels and polymorphisms of the oxytocin receptor gene measured using salivary assays. This represents a movement toward precision medicine.

\section{METHODS AND ANALYSIS \\ Design and trial registration}

This is a multisite, placebo-controlled, triple-blind, sequential, within-subject crossover trial evaluating the efficacy of intranasal oxytocin on pain and function among patients with chronic pain. This is a basket trial consisting of heterogeneous populations of chronic pain conditions from multisites across Canada. This trial will be conducted in compliance with the trial protocol, good clinical practice, institutional ethics boards and applicable regulatory requirements, and is registered on ClinicalTrials.gov. A sample informed consent form can be located in the supplementary file: 'OT and Pain Consent MUN V1.2'.

\section{Study settings}

There is increasing recognition that most common chronic pain conditions are heterogeneous with a high degree of overlap and that most patients enrolled in clinical trials are not representative of community dwelling chronic pain patients. ${ }^{42}$ As such, there will be four participating sites across three provinces, each province recruiting a different primary neuromusculoskeletal (NMSK) pain population. Adults with chronic neuropathic pain were recruited from the Jim Pattison Outpatient Care \& Surgical Centre Pain Clinic (JPOCSC-PC; Surrey) and the Initium Centre for Pain Management (ICPM; Abbotsford). The JPOCSC-PC is a multidisciplinary centre that accepts referrals from a catchment area of 2-million people. Two thousand patients are seen per month, of which approximately $20 \%$ present with chronic neuropathic pain. Consecutive women with chronic pelvic pain will be recruited from the Calgary Chronic Pain Centre (Calgary) and directly from the gynaecology clinics of MR and N-EM. The waitlist for an assessment of chronic pelvic pain is approximately 500 patients. Consecutive adults with chronic musculoskeletal pain will be recruited from the Carbonear General Hospital (Carbonear). Approximately, 40 patients with chronic pain are seen each week, of which approximately $60 \%$ present with primary shoulder, neck or back pain.

\section{Patient eligibility}

Intersite inclusion criteria

(1) Adult (>18 years) males and premenopausal women; (2) on stable medication for pain management for 3 months or more with no anticipated changes during 10 weeks of this trial; (3) moderate pain at baseline (ie, a score of 4-8 on a 10-point Numeric Rating Scale) to prevent floor and ceiling effects; and (4) can commit the use of two forms of effective contraception (eg, barrier methods), or one highly effective method, including abstinence, intrauterine device, intrauterine system, vasectomy, tubal ligation or hormonal contraceptive (eg, combined oral contraceptives, patch, vaginal ring, injectables and implants).

\section{Intersite exclusion criteria}

(1) Positive urine pregnancy test or contemplating pregnancy; (2) concurrent use of another nasal spray; (3) nasal pathology (eg, ears, nose and throat diagnosis); (4) diabetes insipidus; (5) current diagnosis or history of cancer; (6) significant unmanaged psychopathology (eg, severe depression as indicated by a score $\geq 15$ on the Patient Health Questionnaire $9^{43}$ due to its inverse association with patient adherence to procedures ${ }^{44}$; (7) receiving hormone treatment for gender-related motivations; (8) documented cardiovascular event (eg, myocardial infarction); (9) known prolongation of the QTc interval; (10) known hypersensitivity to oxytocin; (11) known latex allergy or (12) known or suspected renal impairment. Exclusion criteria will be vetted through a review of patient medical records and self-report given the concordance between self-report and medical diagnosis. ${ }^{45}$

\section{Intra-site criteria}

Men and women with primary neuropathic pain-pain arising as a direct consequence of a lesion or disease affecting the central or peripheral nervous system ${ }^{46}$-will be eligible. Neuropathic pain will be screened for using a score of $3+$ on the Douleur Neuropathique 4 Interview, ${ }^{47}$ and confirmed on clinical assessment.

\section{Calgary, $A B$}

Women with chronic (intermittent or constant) pelvic musculoskeletal pain (ie, located primarily in the pelvic region and reproducible on palpation of the pelvic floor) who have not received a hysterectomy will be eligible. Women with a primary diagnosis of endometriosis, dysmenorrhoea, functional bowel disorder, interstitial cystitis, fibromyalgia or sacroiliac instability as defined by European Guidelines ${ }^{48}$ will be excluded.

\section{Carbonear, NL}

Men and women with primary musculoskeletal pain of back, neck or shoulder origin will be eligible. Pain will be assessed using the BPI-SF and confirmed through physical examination. ${ }^{49} 50$

\section{Across sites}

Adults with multiple chronic pain conditions will be eligible to participate so long as their primary pain complaint meets eligibility criteria. Recruiting different primary NMSK presentations will allow us to evaluate the generalisability of intranasal oxytocin across common chronic pain presentations typically observed within the community and may allow for improved optimisation of treatment in future work. 


\section{Procedure}

Patient recruitment, screening and enrolment

Potentially eligible patients will be approached by a member of our study team during their regularly scheduled appointment at the clinic, and receive information about the objectives of the trial. Interested patients will undergo screening, including: (1) completion of a urine pregnancy test; (2) blood work to evaluate renal impairment (as defined by an eGFR $<45$ by the Cockcroft-Gault equation), if the history suggests any stage or renal insufficiency, including history of diabetes, inflammatory diseases, hypertension and no creatinine has been drawn in the last 2 years; and (3) ECG to evaluate prolongation of the QTc interval among anyone who may be at risk, including those prescribed antidepressant medication. Patients who meet all eligibility criteria will be randomised to study arm before completing a baseline assessment. Recruitment will begin in September 2021 until the target sample size is recruited, or March 2024.

Randomisation, allocation and concealment

The commercially available software, https://app. studyrandomizer.com/, will be used to generate a list of randomly sequenced numbers for assigning patients to condition in a manner outlined in Consolidated Standards of Reporting Trials (CONSORT) reporting guidelines. $^{5152}$ As depicted in figure 1, patients will be randomised to one of two sequences: (1) 24IU oxytocin, placebo and 48 IU oxytocin; or (2) placebo, 24IU oxytocin and $48 \mathrm{IU}$ oxytocin. Central randomisation stratified by province (British Columbia, Alberta and Newfoundland and Labrador) and performed using a 1:1 allocation schedule with permuted blocks of 4 and 6 . Sex will be added as an additional stratification factor for sites in BC and NL. The lists will be uploaded on a web-based password protected randomisation system. When an eligible participant consents to the study, randomisation website will be contacted through a closed system and randomissation code will be assigned to the participant. Automated audit trails will document the patient allocation number and treatment sequence, and the date and time of transaction.

\section{Blinding}

This is a triple-blind study. In order to protect against expectation effects and biases, neither site investigators

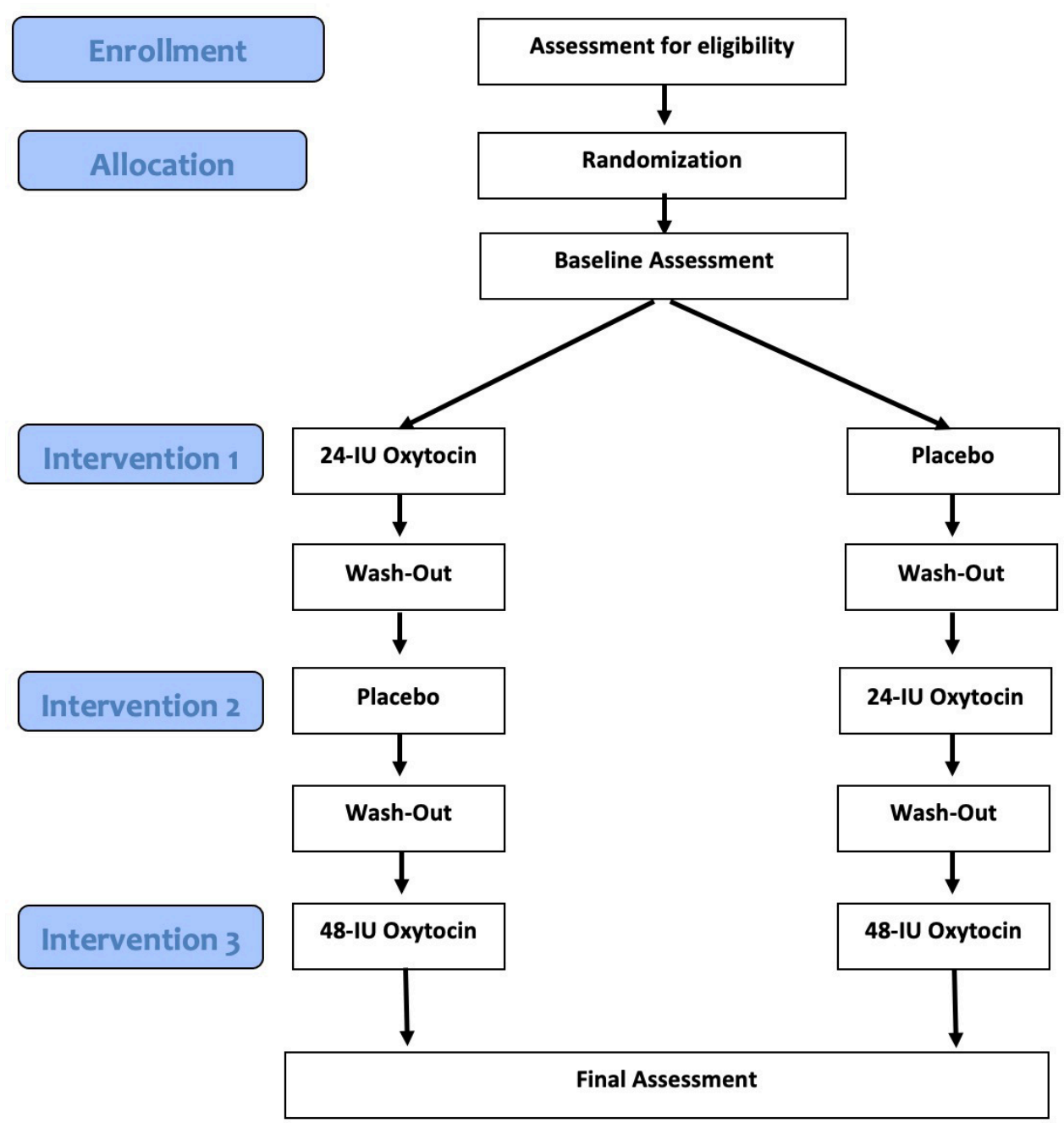

Figure 1 Flow chart of Randomized Controlled Trial design. 


\section{Randomisation}

2-week diaries

\begin{tabular}{|c|c|c|c|c|c|c|}
\hline \multirow[b]{3}{*}{ Testing variables } & \multicolumn{6}{|l|}{ Randomisation } \\
\hline & \multirow[b]{2}{*}{ Phone screen } & \multirow[b]{2}{*}{ Baseline } & \multicolumn{3}{|c|}{ 2-week diaries } & \multirow{2}{*}{$\begin{array}{l}\text { Follow- } \\
\text { up }\end{array}$} \\
\hline & & & Daily diary & Day 7 of diary & Day 14 of diary & \\
\hline Inclusion criteria & $x$ & & & & & \\
\hline Sociodemographics & & $x$ & & & & \\
\hline Medical history & & $x$ & & & & \\
\hline Vitals & & $x$ & & & & $\mathrm{x}$ \\
\hline Pain NRS & $x$ & & $x$ & & & $x$ \\
\hline BPI-SF intensity & & $x$ & & $\mathrm{x}$ & $x$ & \\
\hline BPI-SF interference & & $x$ & & $x$ & $x$ & \\
\hline \multicolumn{7}{|l|}{ Emotional function } \\
\hline Mood & & $x$ & $x$ & & & \\
\hline DASS & & $x$ & & $x$ & $x$ & \\
\hline Sleep & & $x$ & & $x$ & $x$ & \\
\hline Global impression of change & & & & & $x$ & \\
\hline Expectancy & & $x$ & & & & \\
\hline Side effects & & & & & $\mathrm{X}$ & $\mathrm{x}$ \\
\hline
\end{tabular}

BPI-SF, Brief Pain Inventory-Short Form; DASS, Depression, Anxiety and Stress Scale; NRS, Numeric Rating Scale.

nor patients will know which nasal spray contains oxytocin or which sequence of conditions patients are assigned. The allocation sequence will be concealed from researchers using automated randomisation. The bottles containing 24 IU oxytocin, $48 \mathrm{IU}$ oxytocin and placebo will be identical in appearance, smell, texture and taste, and only identifiable through a colour labelling system known to the site pharmacists and study sponsor. Each province's randomisation sequence will be accessed by the site pharmacist and bottles prepared accordingly. Neither the Research Assistant assessing outcomes nor the statistician performing analyses will be unaware of condition. Patient blinding can be broken in the case of an adverse event (eg, emergency department attendance). Patients will be provided a study card with a number to call to reach the central administrator who can de-identify condition in the unlikely case of an adverse drug reaction. The decision to unmask will be made on a case-by-case basis and will depend on potential risk.

\section{Baseline assessment}

Baseline assessments will involve: (1) completion of study measures, refer to table 1 ; (2) collection of approximately $4 \mathrm{~mL}$ of saliva into cryovials using a standard unstimulated passive drool technique. Saliva samples will be frozen until shipped to Salimetrics for analysis of salivary oxytocin concentration and genetic polymorphism in the oxytocin receptor gene OXTR rs53576; and (3) training on procedures for nasal spray administration. Patients will be provided with the option of completing baseline assessments immediately following randomisation, or scheduling a convenient time within 2 weeks. Baseline assessments for women will be scheduled to occur within close proximity to the start of the luteal phase of the menstrual cycle (ie, days 14-28) as this is the stage during which women report greatest pain. ${ }^{53}$ Due to the impact of the global pandemic, patients will have the option of completing study measures virtually. Patients will attend clinic to provide a saliva sample, undergo nasal spray training and receive their assigned nasal spray. Clinic attendance will be scheduled in the afternoon to avoid diurnal fluctuation in saliva oxytocin. Patients will be asked to avoid foods with high sugar, acidity or caffeine 1 hour prior to visiting the clinic as these can confound saliva assays.

\section{Trial interventions}

The intervention will span three 2-week conditions (24IU, $48 \mathrm{IU}$ and placebo) and two 2-week wash-out periods for a total duration of 10 weeks.

\section{Experimental condition}

There will be two experimental conditions. Experimental condition 1: patients will self-administer a 2-week course of 24IU intranasal oxytocin (4IU per puff (12 IU delivered to each nostril); syntocinon, Novartis, Switzerland), twice daily (once in the morning and once in the evening). Experimental condition 2: patients will selfadminister a 2-week course of 48 IU intranasal oxytocin (4IU per puff (24IU delivered to each nostril)), twice daily. Given that treatment adherence is crucial to treatment success, we will ensure standardisation of nasal spray administration by training patients in the selfadministration of intranasal oxytocin in accordance with 
published recommendations. ${ }^{54}$ Intranasal administration of oxytocin is an effective method of administering the neuropeptide across the blood brain barrier. $^{55}$ Twice daily dosing will ensure elevated central concentration of oxytocin throughout the day given that salivary concentration of oxytocin remains elevated for 7 hours following intranasal administration. ${ }^{56}$ Patients will be instructed to store nasal spray at room temperature (between $15^{\circ} \mathrm{C}$ and $25^{\circ} \mathrm{C}$ ) after first use. The inclusion of two doses will allow us to determine the lowest effective dose (ie, 24IU or $48 \mathrm{IU})$, which will inform future trials assessing longterm treatment optimisation. Doses of 24IU and $48 \mathrm{IU}$ were chosen because these are the most frequently used doses in studies with humans and have proven safe for long-term study. ${ }^{49} 50$

\section{Control condition}

Patients will receive an intranasal placebo containing the same ingredients as the oxytocin nasal spray with the exception of active oxytocin. Administration schedule and procedure will be identical to that described in the experimental condition.

\section{Wash-out periods}

Conditions will be separated by a wash-out period of approximately 2 weeks to ensure that oxytocin has fully cleared the system. This time frame is sufficient given that the half-life of oxytocin administered centrally using nasal spray is 2-7 hours and 7 half-lives will be achieved for clearance in 14-49 hours. A 2-week wash-out will also allow each course of intranasal administration to coincide with the same phase of womens' menstrual cycle (ie, the luteal phase). This is relevant given that oestrogen has a priming effect on oxytocin synthesis, release and receptor expression. $^{57}$

\section{Follow-Up}

Patients will be contacted over the phone 2 weeks following completion of the trial (12weeks following randomisation) to assess for potential adverse events.

\section{Patient and public involvement}

Patients with lived experience were consulted in the design of this project and assisted in preparation of study materials. Engagement will continue throughout trial conduction and be emphasised when preparing materials for dissemination.

\section{Measures}

Refer to table 1 for schedule of assessments.

\section{Primary outcome}

The primary outcomes will be the between condition (24 IU vs placebo; $48 \mathrm{IU}$ vs placebo) change in pain intensity and physical function from day 1 to day 14 measured using the BPI-SF. ${ }^{58}$ The BPI-SF measures pain intensity, the impact of pain on seven daily activities (eg, activity, work and sleep) and analgesic use. It has been recommended for use in trials measuring pain across multiple chronic pain conditions. ${ }^{35}$ The BPI-SF was originally designed to measure cancer pain but has been shown to be a reliable and valid instrument for measuring non-cancer pain. ${ }^{59-62}$ Twoweek and 1-month test-retest values for pain and function typically range between 0.72 and $0.98 .^{63}$

\section{Secondary outcomes}

Secondary outcomes include emotional function, sleep disturbance and global impression of change. Emotional function will be measured weekly using the 21-item Depression, Anxiety and Stress Scale. ${ }^{64}$ Scales for depression, anxiety and stress are considered to approximate facets of diagnostic categories, including Depression scale for mood disorders, Anxiety scale for panic disorder and Stress scale for generalised anxiety disorder ${ }^{65}$ Sleep disturbance will be assessed weekly with the Medical Outcomes Study Sleep Scale (MOS-S). ${ }^{66}$ The MOS-S is a 12-item self-report measure designed to assess the important dimensions of sleep, including initiation, maintenance, respiratory problems, quantity, quality and somnolence. Global change across the course of study will be measured at the end of each 2-week course of nasal spray using the Patients' Global Impression of Change (PGIC) Scale. ${ }^{67}$ There has been a wide use of the PGIC Scale in chronic pain trials, ${ }^{68} 69$ and data provide a responsive and readily interpretable measure of participants' assessment of clinical importance of treatment.

\section{Daily diaries}

Ambulatory assessments will be administered by way of REDCap. Patients will complete electronic diaries throughout each 2-week course of nasal spray administration. Patients will make daily recording of: (1) time of nasal spray administration; (2) average pain using a 0 'no pain' to 10 'pain as bad as you can imagine' scale; (3) the degree to which pain has interfered with enjoyment in life and general activity using scales with anchors at 0 'does not interfere' and 10 'interferes completely'. Validated measures will be completed during day 1, day 7 and day 14, refer to table 1. Patients will be asked to guess whether the nasal spray administered contained oxytocin or placebo following each 14-day course of nasal spray administration.

\section{Demographics and covariates}

A demographic questionnaire will collect age, ethnicity, medical comorbidities, medications taken, employment status, marital status, obstetrical history, menstrual history, urinary symptoms, headaches, substance use and smoking status. Concern over pain will be assessed using the 13-item Pain Catastrophizing Scale. ${ }^{70}$ Treatment expectations will be measured with the Credibility/ Expectancy Questionnaire. ${ }^{71}$ Allodynia/hyperalgesia will be assessed using the Central Sensitisation Inventory ${ }^{72} 73$ and confirmed with Q-tip testing. ${ }^{74}$

Side effects and safety monitoring

As recommended for trials of chronic pain, ${ }^{75}$ side effects will be assessed using open-ended prompts (ie, have 
you experienced any unwanted symptoms in the past 24 hours?) and the Symptom Assessment Schedule ${ }^{76}$ supplemented with additional symptoms (eg, euphoria, nasal irritation and dizziness) identified in a recent trial evaluating the effect of intranasal oxytocin on pain in a sample of 14 women with fibromyalgia. ${ }^{34}$

\section{Sample size calculation}

We based our sample size calculation on the number of patients needed to evaluate a clinically significant reduction in pain intensity and improvement in physical function of the primary outcomes: change in pain intensity and physical function from day 1 to day 14 that occurs between conditions (24IU oxytocin vs placebo; $48 \mathrm{IU}$ oxytocin vs placebo). The hypothesis tests are one sided and the trial is powered using a sequential, repeatedmeasures crossover design with one interim analysis to monitor the preliminary activity of the $24 \mathrm{IU}$ and $48 \mathrm{IU}$ doses of oxytocin relative to placebo (refer to section 3.2.1 for greater detail on the interim analysis).

The overall type I error rate will be 0.05 and the O'Brien and Fleming's method ${ }^{77}$ will be used to control for the inflation of type I error due to the interim analysis. The interim analysis will be conducted when primary outcomes are available for half of the proposed sample. The Sidak method ${ }^{78}$ will be used to adjust for multiple comparisons (ie, $\alpha=0.0127$ after adjusting for 4 comparisons: 24IU and 48 IU doses of oxytocin compared with placebo for pain intensity and physical function). Based on previous research with chronic pain populations, ${ }^{58}$ we assume a SD of 2.0 for pain intensity and physical function and a block-based symmetric correlation matrix under which the 2-week correlation within the same arm is assumed to be $0.8^{63}$; the subcorrelation matrix of day 1 and day 14 pain intensity and physical function across two arms is comparable for any two arms; and the correlation of day 1 and day 1 on two arms equals that of day 14 and day 14. We anticipate that the correlation of day 1 and day 14 cross-arms will be lower than the correlation of the same day pain intensity/physical function. As a conservative measure, we assume the correlation of day 1 and day 14 is comparable to the same day correlation cross two arms and a better power will be achieved if the correlation between day 1 and day 14 is lower. The sample size is determined as the largest for two scenarios on whether the subcorrelation matrix of day 1 and day 14 is stable over phases or not.

For the scenario of stable subcorrelation matrix of day 1 and day 14, all phases data will be included in the analysis. Target power is 0.9 to detect a $0.5 \mathrm{SD}$ difference in pain intensity/physical function, which corresponds to an effect size of 0.56 for the outcomes of change between day 1 and day 14 under the aforementioned assumptions on the covariance matrix. The required sample size is 41 per province to accommodate one interim analysis. If the subcorrelation matrix of day 1 and day 14 is unstable cross phases, only the first two phases data will be included in the analysis and the size for evaluating the efficacy of the
$24 \mathrm{IU}$ and $48 \mathrm{IU}$ over the placebo is reduced to $2 / 3$, where the two samples are independent ( $\alpha=0.0127$ using Sidak). Under this scenario, the power is set as 0.8 for the same effect size and the required sample size is 54 , which is increased to 81 accounting for abandoning the phase III data due to instability of the correlation. Therefore, the sample size of 81 per province will be used and this is the robust size to guarantee at least $80 \%$ power and an overall type I error of 0.05 with an interim analysis for different scenarios of the correlation matrix of pain intensity/physical function. The covariates of sex and province will be adjusted and the rule of 8 additional cases per covariate will be followed for the combined analysis on the efficacy of $24 \mathrm{IU}$ and $48 \mathrm{IU}$ oxytocin relative to placebo. Accounting for a potential attrition rate of $20 \%$ and the balance among the 2 sequences per province, a total sample of 336 patients (112 per province) is required.

\section{Data analysis}

An intention-to-treat (ITT) analysis will be performed, ${ }^{79}$ in accordance with recommendations by CONSORT. ${ }^{51} 52$ ITT analyses provide an assessment of the practical impact of the treatment. Primary analyses: the hypotheses that improvement in pain and physical function will be observed following 2-week administration of oxytocin nasal spray relative to placebo will be assessed using BPI-SF average 24-hour pain intensity scores. Using linear mixed models in SAS, the analytic strategy will be a mixed models analysis of covariance with fixed effects (time (day 1 and day 14) and condition (24 IU oxytocin, 48 IU oxytocin and placebo)) and random effect (individual), and sex (male and female) as between-subject factor after adjusting for relevant covariates (ie, province, basal oxytocin at baseline, expectation, pain catastrophising and medical comorbidity). One interim analysis will be performed (see 2.17 for details). Based on previous literature, BPI-SF assessed pain is a relatively 'well-behaved' variable with respect to the assumptions of a general linear model ${ }^{80}$ Even so, prior to conducting any analyses, preliminary examination of the assumptions of the General Linear Model will be conducted. ${ }^{80}$ Should the data indicate violation of assumption, we will perform appropriate transformations and/or consider the appropriate interaction term(s) ${ }^{80}$ Missing data will be handled using multiple imputation ${ }^{81}$ in accordance to Harrell's guidelines. ${ }^{82}$ Secondary analyses: the effects of the intervention on change in emotional function, sleep disturbance and global impression of change between conditions will be evaluated in a manner analogous to that described above. Exploratory analyses will be performed to evaluate whether basal oxytocin concentration or polymorphism of the oxytocin gene receptor moderate or mediate treatment outcomes.

\section{Frequency of analysis}

An interim analysis will be conducted when complete data are available from half of the desired sample size. The interim analysis will be used to decide: (1) stop the 
trial for efficacy if overwhelming evidence indicates that the $48 \mathrm{IU}$ dose of intranasal oxytocin is efficacious relative to placebo ( $p$ value $<0.0083$ ); (2) drop the 24 IU dose for inefficacy if evidence supports a conservative test of the null hypothesis that 24 IU oxytocin is equivalent to placebo if the conditional power is less than 0.2 if continuing the stage 2 data collection; or (3) continue the trial as planned. The O'Brien and Fleming's method will control the overall type I rate at 0.0127 with 0.0083 $\alpha$ spending at the interim, and the overall type II error rate at 0.1 with 0.029 spending at the interim for each of the $24 \mathrm{IU}$ and $48 \mathrm{IU}$ doses and primary outcome. Final analyses will commence after the follow-up has been completed for the final participant enrolled (eg, May 2024) and span 3 months in duration.

\section{Subgroup analyses}

Subgroup analyses (eg, dose of oxytocin, province and sex) are built into the analytic plan. Secondary analyses include interactions between dose, pain type, sex and gender.

\section{Risk management and safety monitoring board}

A Data and Safety Monitoring Board (DSMB) has been established to perform (unblinded) analyses according to the DSMB charter. The DSMB is composed of three independent clinical experts, and a statistician with expertise in Randomized Controlled Trials. The DSMB will advise on any serious adverse event reported during the conduct of the trial. Moreover, safety and efficacy will be evaluated during the interim analysis. Early termination will only occur if the DSMB is unanimous in their conclusion of an unfavourable benefit-to-risk ratio. ${ }^{83}$ Strict termination criteria were not established a priori given that: (1) rescue medication is not needed for intranasal oxytocin. The best method to resolve side effects is to discontinue use of the drug, which is our recommended course of action; (2) chronic NMSK pain is not a life-threatening condition; (3) study duration is brief; and (4) intranasal oxytocin has been extensively studied and side effects are rare and benign. ${ }^{84}$ The advice of the DSMB will be sent to the study sponsor. Should the sponsor decide not to fully implement the advice of the DSMB, the sponsor will send the advice to the reviewing medical research ethics committee, including a note to substantiate why (part of) the advice of the DSMB will not be followed.

\section{Ethics and dissemination}

Results from this feasibility trial will be disseminated to the academic community through conference presentations and the publication of peer-reviewed manuscripts. Results will be posted to our website www.munbehaviourmedicine.ca and made available to patients, providers and the general public. Trial protocols were approved by the Newfoundland and Labrador Health Research Ethics Board (HREB \#20227), University of Calgary Conjoint Health Research Ethics Board (REB20 \#0359), University of British Columbia Clinical Research Ethics Board
(CREB \#H20-00729) and Health Canada (Control \# 252780).

\section{Data management}

Data will be collected, de-identified and stored. Electronic data will be stored on password-protected servers in encrypted files. De-identified data will be retained for 25 years and made available to members of the investigative team. Individual participant de-identified data (including data dictionaries) will be made available beginning 3 months after final follow-up data has been collected (anticipated September 2024) to researchers who provide a methodologically sound proposal for the purpose of achieving the aims of the approved proposal. Data sharing will be enacted with a data-transfer agreement between the sending and receiving institutions. Proposals should be directed to the corresponding author (JR).

\section{Patient adherence}

We will use a multi-pronged approach to encourage patient engagement. First, the timeline and demands of the trial will be explicitly discussed at the outset with patients, who will be asked to sign a behavioural contract to commit to trying to meet the requirements. Second, patients will receive telephone reminders prior to each laboratory visit and again if they miss one visit. Third, expectations will be developed for attendance. Patients will know our research staff by name and made aware that their research associate has an appointment scheduled with them and will be awaiting their arrival. Fourth, patients who have difficulties attending sessions will be provided with a motivational conversation during which ambivalence towards attending sessions will be openly discussed with the goal of securing commitment to attend sessions. We have successfully employed these strategies with some very challenging patient groups (eg, patients with cancer-related fatigue and obese patients attending exercise sessions). These strategies have been identified by Cochrane reviews as methods for improving patient recruitment ${ }^{85}$ and retention. ${ }^{86}$

\section{Author affiliations}

${ }^{1}$ Department of Psychology, Faculty of Science, Memorial University of Newfoundland, St. John's, Newfoundland, Canada

${ }^{2}$ Department of Psychology, Faculty of Arts, University of Calgary, Calgary, Alberta, Canada

${ }^{3}$ Canadian Injured Workers Alliance, Thunder Bay, Ontario, Canada

${ }^{4}$ Anesthesia and Pain Medicine, Faculty of Medicine, Memorial University of Newfoundland, St. John's, Newfoundland and Labrador, Canada

${ }^{5}$ Anesthesiology, Pharmacology and Therapeutics, Faculty of Medicine, University of British Columbia, Vancouver, British Columbia, Canada

${ }^{6}$ Department of Anesthesiology, Perioperative and Pain Medicine, Cumming School of Medicine, University of Calgary, Calgary, Alberta, Canada

${ }^{7}$ Obstetrics and Gynecology, Cumming School of Medicine, University of Calgary, Calgary, Alberta, Canada

${ }^{8}$ The Ottawa Hospital Research Institute, Ottawa, Ontario, Canada

${ }^{9}$ Department of Anesthesiology and Pain Medicine, University of Ottawa, Ottawa,

Ontario, Canada

${ }^{10}$ The Ottawa Hospital Pain Clinic, Ottawa, Ontario, Canada 
${ }^{11}$ Community Health and Humanities, Faculty of Medicine, Memorial University of Newfoundland, St. John's, Newfoundland, Canada

Contributors All authors (JR, TC, LC, DF, AM, AAM, N-EM, PP, MR and YY) were involved in the conceptualisation and design of the trial, made significant intellectual contributions to the written protocol and have approved the submitted version.

Funding This work was supported by the Canadian Institutes of Health Research (grant number: 426528). The funder was not involved in trial design, conduct or reporting.

Competing interests None declared.

Patient consent for publication Not applicable.

Provenance and peer review Not commissioned; peer reviewed for ethical and funding approval prior to submission.

Data availability statement Data sharing not applicable as no datasets generated and/or analysed for this study. Individual participant de-identified data (including data dictionaries) will be made available beginning 3-months after final follow-up data has been collected (anticipated September 2024) to researchers who provide a methodologically sound proposal for the purpose of achieving the aims of the approved proposal. Data sharing will be enacted with a data-transfer agreement between the sending and receiving institutions. Proposals should be directed to Joshua Rash (jarash@mun.ca)

Supplemental material This content has been supplied by the author(s). It has not been vetted by BMJ Publishing Group Limited (BMJ) and may not have been peer-reviewed. Any opinions or recommendations discussed are solely those of the author(s) and are not endorsed by BMJ. BMJ disclaims all liability and responsibility arising from any reliance placed on the content. Where the content includes any translated material, BMJ does not warrant the accuracy and reliability of the translations (including but not limited to local regulations, clinical guidelines, terminology, drug names and drug dosages), and is not responsible for any error and/or omissions arising from translation and adaptation or otherwise.

Open access This is an open access article distributed in accordance with the Creative Commons Attribution Non Commercial (CC BY-NC 4.0) license, which permits others to distribute, remix, adapt, build upon this work non-commercially, and license their derivative works on different terms, provided the original work is properly cited, appropriate credit is given, any changes made indicated, and the use is non-commercial. See: http://creativecommons.org/licenses/by-nc/4.0/.

\section{ORCID iDs}

Joshua A. Rash http://orcid.org/0000-0003-0927-0712

Anastasia A. Mekhael http://orcid.org/0000-0003-0620-3872

\section{REFERENCES}

1 Merskey $\mathrm{H}$. Pain terms: a list with definitions and notes on usage. Recommended by the International association for the study of pain. Pain 1979;6:249-52.

2 IoM IOM. Relieving pain in America: a blueprint for transforming prevention, care, education, and research. Washington DC: National Academy of Sciences, 2011.

3 Schopflocher D, Taenzer P, Jovey R. The prevalence of chronic pain in Canada. Pain Res Manag 2011;16:445-50.

4 Stanford EA, Chambers CT, Biesanz JC, et al. The frequency, trajectories and predictors of adolescent recurrent pain: a population-based approach. Pain 2008;138:11-21.

5 Hadjistavropoulos T, Marchildon GP, Fine PG, et al. Transforming long-term care pain management in North America: the policyclinical interface. Pain Med 2009;10:506-20.

6 Turk DC, Wilson HD, Cahana A. Treatment of chronic non-cancer pain. Lancet 2011;377:2226-35

7 Martin BI, Deyo RA, Mirza SK, et al. Expenditures and health status among adults with back and neck problems. JAMA 2008;299:656-64.

8 Busse JW, Wang L, Kamaleldin M, et al. Opioids for chronic noncancer pain: a systematic review and meta-analysis. JAMA 2018;320:2448-60.

9 Gregorian RS, Gasik A, Kwong WJ, et al. Importance of side effects in opioid treatment: a trade-off analysis with patients and physicians. J Pain 2010;11:1095-108.

10 Sostres C, Gargallo CJ, Arroyo MT, et al. Adverse effects of nonsteroidal anti-inflammatory drugs (NSAIDs, aspirin and coxibs) on upper gastrointestinal tract. Best Pract Res Clin Gastroenterol 2010;24:121-32.

11 González-Hernández A, Rojas-Piloni G, Condés-Lara M. Oxytocin and analgesia: future trends. Trends Pharmacol Sci 2014;35:549-51.

12 Rash JA, Aguirre-Camacho A, Campbell TS. Oxytocin and pain: a systematic review and synthesis of findings. Clin J Pain 2014;30:453-62.

13 Tracy LM, Georgiou-Karistianis N, Gibson SJ, et al. Oxytocin and the modulation of pain experience: implications for chronic pain management. Neurosci Biobehav Rev 2015;55:53-67.

14 Rash JA, Toivonen K, Robert M, et al. Protocol for a placebocontrolled, within-participants crossover trial evaluating the efficacy of intranasal oxytocin to improve pain and function among women with chronic pelvic musculoskeletal pain. BMJ Open 2017; 7:e014909.

15 Viero C, Shibuya I, Kitamura N, et al. Review: oxytocin: crossing the bridge between basic science and pharmacotherapy. CNS Neurosci Ther 2010;16:e138-56.

16 Gimpl G, Fahrenholz F. The oxytocin receptor system: structure, function, and regulation. Physiol Rev 2001;81:629-83.

17 Breton J-D, Veinante P, Uhl-Bronner S, et al. Oxytocin-induced antinociception in the spinal cord is mediated by a subpopulation of glutamatergic neurons in lamina I-II which amplify GABAergic inhibition. Mol Pain 2008;4:19.

18 Condés-Lara M, Rojas-Piloni G, Martínez-Lorenzana G, et al. Hypothalamospinal oxytocinergic antinociception is mediated by GABAergic and opiate neurons that reduce A-delta and $C$ fiber primary afferent excitation of spinal cord cells. Brain Res 2009;1247:38-49.

19 Jo YH, Stoeckel ME, Freund-Mercier MJ, et al. Oxytocin modulates glutamatergic synaptic transmission between cultured neonatal spinal cord dorsal horn neurons. J Neurosci 1998;18:2377-86.

$20 \mathrm{Ge}$ Y, Lundeberg T, Yu L-C. Blockade effect of mu and kappa opioid antagonists on the anti-nociception induced by intraperiaqueductal grey injection of oxytocin in rats. Brain Res 2002;927:204-7.

21 Yang J, Liang J-Y, Li P, et al. Oxytocin in the periaqueductal gray participates in pain modulation in the rat by influencing endogenous opiate peptides. Peptides 2011;32:1255-61.

22 Miranda-Cardenas Y, Rojas-Piloni G, Martínez-Lorenzana G, et al. Oxytocin and electrical stimulation of the paraventricular hypothalamic nucleus produce antinociceptive effects that are reversed by an oxytocin antagonist. Pain 2006;122:182-9.

23 Berna C, Leknes S, Holmes EA, et al. Induction of depressed mood disrupts emotion regulation neurocircuitry and enhances pain unpleasantness. Biol Psychiatry 2010;67:1083-90.

24 Rainville P, Bao QVH, Chrétien P. Pain-related emotions modulate experimental pain perception and autonomic responses. Pain 2005;118:306-18.

25 Rhudy JL, Meagher MW. Fear and anxiety: divergent effects on human pain thresholds. Pain 2000;84:65-75.

26 Heinrichs M, Baumgartner T, Kirschbaum C, et al. Social support and oxytocin interact to suppress cortisol and subjective responses to psychosocial stress. Biol Psychiatry 2003;54:1389-98.

27 Uryvaev YV, Petrov G. Extremely low doses of oxytocin reduce pain sensitivity in men. Bull Exp Biol Med 1996;122:1071-3.

28 Singer T, Snozzi R, Bird G, et al. Effects of oxytocin and prosocial behavior on brain responses to direct and vicariously experienced pain. Emotion 2008;8:781.

29 Rash JA, Campbell TS. The effect of intranasal oxytocin administration on acute cold pressor pain: a placebo-controlled, double-blind, within-participants crossover investigation. Psychosom Med 2014;76:422-9.

30 Yang J. Intrathecal administration of oxytocin induces analgesia in low back pain involving the endogenous opiate peptide system. Spine 1994;19:867-71.

31 Wang Y-L, Yuan Y, Yang J, et al. The interaction between the oxytocin and pain modulation in headache patients. Neuropeptides 2013;47:93-7.

32 Ohlsson B, Truedsson M, Bengtsson M, et al. Effects of longterm treatment with oxytocin in chronic constipation; a double blind, placebo-controlled pilot trial. Neurogastroenterol Motil 2005;17:697-704.

33 Louvel D, Delvaux M, Felez A, et al. Oxytocin increases thresholds of colonic visceral perception in patients with irritable bowel syndrome. Gut 1996;39:741-7.

34 Mameli S, Pisanu GM, Sardo S, et al. Oxytocin nasal spray in fibromyalgic patients. Rheumatol Int 2014;34:1047-52.

35 Dworkin RH, Turk DC, Farrar JT, et al. Core outcome measures for chronic pain clinical trials: IMMPACT recommendations. Pain 2005;113:9-19. 
36 Alfvén G. Plasma oxytocin in children with recurrent abdominal pain. $J$ Pediatr Gastroenterol Nutr 2004;38:513-7.

37 Alfvén G, de la Torre B, Uvnäs-Moberg K. Depressed concentrations of oxytocin and cortisol in children with recurrent abdominal pain of non-organic origin. Acta Paediatr 1994;83:1076-80.

38 Domes G, Heinrichs M, Gläscher J, et al. Oxytocin attenuates amygdala responses to emotional faces regardless of valence. Biol Psychiatry 2007;62:1187-90.

39 Kirsch P, Esslinger C, Chen Q, et al. Oxytocin modulates neural circuitry for social cognition and fear in humans. $J$ Neurosci 2005;25:11489-93.

40 Rodrigues SM, Saslow LR, Garcia N, et al. Oxytocin receptor genetic variation relates to empathy and stress reactivity in humans. Proc Natl Acad Sci U S A 2009;106:21437-41.

41 Chen FS, Kumsta R, von Dawans B, et al. Common oxytocin receptor gene (OXTR) polymorphism and social support interact to reduce stress in humans. Proc Natl Acad Sci U S A 2011;108:19937-42.

42 Maixner W, Fillingim RB, Williams DA, et al. Overlapping chronic pain conditions: implications for diagnosis and classification. J Pain 2016;17:T93-107.

43 Kroenke K, Spitzer RL, Williams JB. The phq-9. J Gen Intern Med 2001;16:606-13.

44 DiMatteo MR, Lepper HS, Croghan TW. Depression is a risk factor for noncompliance with medical treatment: meta-analysis of the effects of anxiety and depression on patient adherence. Arch Intern Med 2000;160:2101-7.

45 Kehoe R, Wu SY, Leske MC, et al. Comparing self-reported and physician-reported medical history. Am J Epidemiol 1994;139:813-8.

46 Treede R-D, Jensen TS, Campbell JN, et al. Neuropathic pain: redefinition and a grading system for clinical and research purposes. Neurology 2008;70:1630-5.

47 Bouhassira D, Attal N, Alchaar $\mathrm{H}$, et al. Comparison of pain syndromes associated with nervous or somatic lesions and development of a new neuropathic pain diagnostic questionnaire (DN4). Pain 2005;114:29-36.

48 Vleeming A, Albert HB, Ostgaard HC, et al. European guidelines for the diagnosis and treatment of pelvic girdle pain. Eur Spine $J$ 2008;17:794-819.

49 Association AP. Diagnostic and statistical manual of mental disorders (DSM-5). 5th edn. Arlington, VA: American Psychiatric Association, 2013.

50 Turk DC, Melzack R. Handbook of pain assessment. 3rd edn. New York, NY: Guilford Press, 2011.

51 Moher D, Hopewell S, Schulz KF, et al. CONSORT 2010 explanation and elaboration: updated guidelines for reporting parallel group randomised trials. Int J Surg 2012;10:28-55.

52 Schulz KF, Altman DG, Moher D, et al. CONSORT 2010 statement: updated guidelines for reporting parallel group randomised trials. PLoS Med 2010;7:e1000251.

53 Riley JL, Robinson ME, Wise EA, et al. A meta-analytic review of pain perception across the menstrual cycle. Pain 1999;81:225-35.

54 Guastella AJ, Hickie IB, McGuinness MM, et al. Recommendations for the standardisation of oxytocin nasal administration and guidelines for its reporting in human research. Psychoneuroendocrinology 2013;38:612-25.

55 Born J, Lange T, Kern W, et al. Sniffing neuropeptides: a transnasal approach to the human brain. Nat Neurosci 2002;5:514-6.

56 van ljzendoorn $\mathrm{MH}$, Bhandari R, van der Veen $\mathrm{R}$, et al. Elevated Salivary Levels of Oxytocin Persist More than $7 \mathrm{~h}$ after Intranasal Administration. Front Neurosci 2012;6:174.

57 Light KC, Grewen KM, Amico JA, et al. Oxytocinergic activity is linked to lower blood pressure and vascular resistance during stress in postmenopausal women on estrogen replacement. Horm Behav 2005; $47: 540-8$

58 Cleeland CS, Ryan KM. Pain assessment: global use of the brief pain inventory. Ann Acad Med Singap 1994;23:129-38.

59 Keller S, Bann CM, Dodd SL, et al. Validity of the brief pain inventory for use in documenting the outcomes of patients with noncancer pain. Clin J Pain 2004;20:309-18.
60 Mendoza T, Mayne T, Rublee D, et al. Reliability and validity of a modified brief pain inventory short form in patients with osteoarthritis. Eur J Pain 2006;10:353-61.

61 Tan G, Jensen MP, Thornby Jl, et al. Validation of the brief pain inventory for chronic nonmalignant pain. J Pain 2004;5:133-7.

62 Kapstad H, Rokne B, Stavem K. Psychometric properties of the brief pain inventory among patients with osteoarthritis undergoing total hip replacement surgery. Health Qual Life Outcomes 2010;8:148.

63 Cleeland C. The brief pain inventory: user guide. University of Texas, TX: M. D. Anderson Cancer Center, 2009

64 Lovibond P. Manual for the depression anxiety stress scales. Sydney: Sydney Psychology edition, 1995.

65 Brown TA, Chorpita BF, Korotitsch W, et al. Psychometric properties of the depression anxiety stress scales (DASS) in clinical samples. Behav Res Ther 1997;35:79-89.

66 Hays RD, Stewart A. Sleep measures, 1992.

67 Hurst $\mathrm{H}$, Bolton J. Assessing the clinical significance of change scores recorded on subjective outcome measures. J Manipulative Physiol Ther 2004;27:26-35.

68 Dunkl PR, Taylor AG, McConnell GG, et al. Responsiveness of fibromyalgia clinical trial outcome measures. J Rheumatol 2000;27:2683-91.

69 Farrar JT, Young JP, LaMoreaux L, et al. Clinical importance of changes in chronic pain intensity measured on an 11-point numerical pain rating scale. Pain 2001;94:149-58.

70 Sullivan MJ, Bishop SR, Pivik J. The pain catastrophizing scale: development and validation. Psychol Assess 1995;7:524.

71 Devilly GJ, Borkovec TD. Psychometric properties of the credibility/expectancy questionnaire. J Behav Ther Exp Psychiatry 2000;31:73-86

72 Mayer TG, Neblett R, Cohen H, et al. The development and psychometric validation of the central sensitization inventory. Pain Pract 2012;12:276-85.

73 Neblett R, Hartzell MM, Mayer TG, et al. Establishing clinically relevant severity levels for the central sensitization inventory. Pain Pract 2017:17:166-75.

74 Nasr-Esfahani M, Jarrell J. Cotton-tipped applicator test: validity and reliability in chronic pelvic pain. Am J Obstet Gynecol 2013;208:52 e1-5.

75 Katz NP. The measurement of symptoms and side effects in clinical trials of chronic pain. Contemp Clin Trials 2012;33:903-11.

76 Kristjanson L, Pickstock S, Yuen K. Development and testing of the revised symptom assessment scale (SAS): final report. Perth, Western Australia: Edith Cowan University, 1999.

77 O'Brien PC, Fleming TR. A multiple testing procedure for clinical trials. Biometrics 1979;35:549-56.

78 idák Z. Rectangular confidence regions for the means of multivariate normal distributions. J Am Stat Assoc 1967;62:626-33.

79 Montori VM, Guyatt GH. Intention-to-treat principle. CMAJ 2001;165:1339-41.

80 Tabachnick BG, Fidell LS. Using multivariate statistics. 6th edn. Boston, MA: Pearson, 2012

81 Little RJ, Rubin DB. Statistical analysis with missing data. statistical analysis with missing data, 2nd ED, by RJA little and DB Rubin Wiley series in probability and stistics. New York, NY: Wiley, 2002, ;: 12002.

82 Harrell F. Regression modeling strategies: with applications to linear models, logistic and ordinal regression, and survival analysis. New York, NY: Springer, 2001

83 Ellenberg SS, Fleming TR, DeMets DL. Data monitoring committees in clinical trials: a practical perspective. West Sussex, England: John Wiley \& Sons, Ltd, 2002.

84 MacDonald E, Dadds MR, Brennan JL, et al. A review of safety, side-effects and subjective reactions to intranasal oxytocin in human research. Psychoneuroendocrinology 2011:36:1114-26.

85 Treweek S, Lockhart P, Pitkethly M, et al. Methods to improve recruitment to randomised controlled trials: cochrane systematic review and meta-analysis. BMJ Open 2013;3 doi:10.1136/ bmjopen-2012-002360

86 Caldwell PHY, Hamilton S, Tan A, et al. Strategies for increasing recruitment to randomised controlled trials: systematic review. PLOS Med 2010;7:e1000368. 